STRUCTURE AND DYNAMICS OF ATOMS AND MOLECULES: CONCEPTUAL TRENDS 
This book is a companion volume to

Conceptual Trends in Quantum Chemistry,

edited by E. S. Kryachko and J. L. Calais, ISBN 0-7923-2621-0 


\title{
Structure and Dynamics of
}

\section{Atoms and Molecules: Conceptual Trends}

\author{
Edited by \\ J. L. Calais \\ Quantum Theory Project, \\ University of Florida, Gainesville, U.S.A. \\ and \\ E. S. Kryachko \\ The Bogoliubov Instifute for Theoretical Physics, \\ Kiev, Ukraine
}

SPRINGER SCIENCE+BUSINESS MEDIA, B.V. 
Library of Congress Cataloging-in-Publication Data

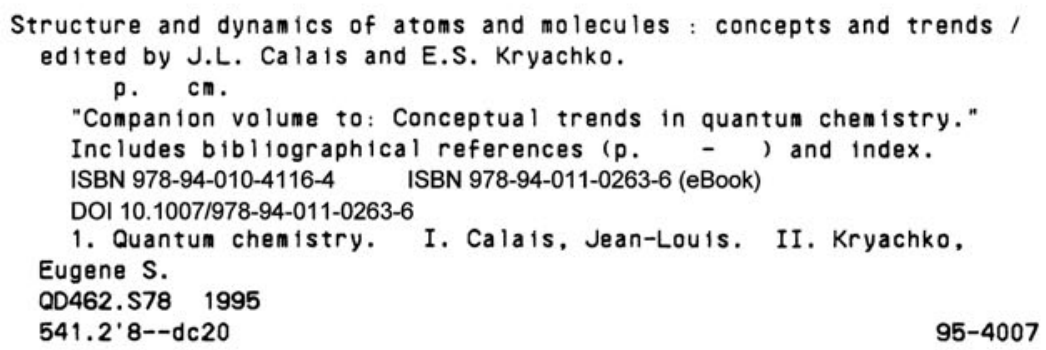

\section{Printed on acid-free paper}

\section{All Rights Reserved}

(C) 1995 Springer Science+Business Media Dordrecht

Originally published by Kluwer Academic Publishers in 1995

Softcover reprint of the hardcover 1st edition 1995

No part of the material protected by this copyright notice may be reproduced or utilized in any form or by any means, electronic or mechanical, including photocopying, recording or by any information storage and retrieval system, without written permission from the copyright owner. 


\section{CONTENTS}

Foreword

Elements of Hydrogen Transfer Theory

R. Lefebvre

1. Introduction

2. One-Dimensional Tunneling

3. Spectroscopic Splittings

3.1. 1-Dimensional Treatments

3.2. N-Dimensional Treatments

4. From Quantum Beats to Relaxation

5. Calculation of Relaxation Rates

References

Building a Bridge Between Ab Initio and Semiempirical Theories of Molecular Electronic Structure

K. F. Freed

1. Introduction

2. Theoretical Bridge Between Ab Initio and Semiempirical Methods

2.1. Derivation of the Exact Effective Valence

Shell Only Equations

2.2. Properties of and Computational Methods for $\mathrm{H}^{\mathrm{V}}$

3. The $\mathrm{Ab}$ Initio $\mathrm{H}^{\mathrm{V}}$ Method 
vi

3.1. Description of the $\mathrm{Ab}$ Initio $\mathrm{H}^{\mathrm{V}}$ Method 37

3.2. Illustrations of Recent $\mathrm{Ab}$ Initio $\mathrm{H}^{\mathrm{V}}$ Calculations 43

4. Ab Initio Computations of Correlated Semiempirical-Like Integrals 49

4.1. Computations of One-Center $\mathrm{H}^{\mathrm{V}}$ Parameters for Atomic Systems 53

4.2. Ab Initio Computation of Parameters in Pi-Electron Methods 56

4.3. Ab Initio Computations of the Effective Dipole

Operator: Comparisons with Semiempirical Methods $\quad 62$

5. Acknowledgment 64

6. References 64

Electron Delocalization in The Theory of Intermolecular and Intergroup Interactions: Cause, Effect, Prevention

W. H. Adams

1. Introduction 69

2. Unphysical States 71

3. Separability and Localizability of Electronic Wave Functions 73

3.1. Minimally Distorted Localized Wave Functions 74

3.2. Genuine Primitive Functions 78

$\begin{array}{ll}\text { 4. Level Shift Operators } & 79\end{array}$

5. Hartree-Fock Approximation $\quad 82$

5.1. Localized Orbital Equations 83

5.2. Huzinaga-Equation 87

6. Correspondences and Extensions $\quad 89$

$\begin{array}{ll}\text { Appendix A } & 92\end{array}$

References $\quad 93$ 
Quantum Dynamics of Diatoms in External Fields

J. Broeckhove, B. Feyen, and P. Van Leuven

1. Introduction $\quad 97$

2. The Hamiltonian 98

2.1. Born-Oppenheimer and Beyond 99

2.2. The Dipole Interaction 101

2.3. Diatoms in the Dipole Field 102

3. Quantum Time Evolution 104

4. Multiphoton Absorption in Hydrogen Fluoride 108

4.1. Vibrational Motion 109

4.2. Rovibrational Motion 113

5. Laser Assisted Dynamics in Boron Hydride 114

5.1. The Excitation Pathway 119

5.2. The Vibronic Picture 120

5.3. The Rovibronic Picture 125

6. Conclusions 127

$\begin{array}{ll}\text { References } & 129\end{array}$

Dimensional Scaling in Quantum Theory

J. Avery

1. Introduction 133

2. The Jacobian Weighting Factor 135

3. The Large-D Limit 138

4. Three-Particle Systems 139

5. An Atom-to-Molecule Transformation 142

6. N-Particle Systems 148

7. Discussion 151

Acknowledgements 151

References 152 
viii

Probing The Collective and Independent-Particle Character of Atomic Electrons

R. S. Berry

1. Introduction 155

1.1. Historical Background 155

1.2. Alternative Quantizations and Constants of Motion 156

1.3. The Tools of the Analysis 158

2. Symmetry-Based Interpretation 159

2.1. Initial Explorations 159

2.2. Multiplets and Supermultiplets 160

3. Interpretations Based on Wave Functions 162

3.1. Graphic Representations of Helium 162

3.2. Graphical Representations for Other Atoms: Alkaline Earths 163

3.3. Overlap Criteria 167

3.4. Oscillator Strength Criteria 168

3.5. Quadrupole Moments 168

3.6. Angular Correlations in Double Ionization 171

3.7. Other Correlations and Dispersions 172

4. Interpretations Based on Classical and Semiclassical Models 173

4.1. The Bohr-Sommerfeld Approach 173

4.2. Orbits, Planetary Atoms, Langmuir States 174

5. Summary and Conclusions 176

$\begin{array}{ll}\text { References } & 176\end{array}$

Electronic Structure Models: Computations, Chemical Insights and Appropriateness

1. Introduction 184

2. Model Hamiltonians

3. Response Properties, Wave Functions, and Levels of Theory 186

$\begin{array}{ll}\text { 4. Example: The Frequency Dependent Hyperpolarizabilities of Push Pull } & \\ \text { Substituted Aromatics } & 191\end{array}$

A. Model Hamiltonian 191

B. Ground State 192

C. Nature of the Excitations 192

D. Some Selected Results 193

$\begin{array}{ll}\text { References } & 208\end{array}$ 


\section{The Work Formalism: A New Theory of Electronic Structure}

1. Introduction

2. The Work Formalism 216

2.1. General Framework 216

2.2. The Work Formalism Hartree Approximation 220

2.3. The Work Formalism Hartree-Fock Approximation 222

2.4. The Work Formalism Configuration-Interaction Approximation 225

2.5. Asymptotic Structure of Exchange-Correlation Potential 226

3. Comparisons with Other Local Potential Theories 227

3.1. Comparison with Kohn-Sham Theory 227

3.2. Rigorous Interpretation of the Local Density Approximation 232

3.3. Comparison with Slater Theory 237

4. Results of Application to Atoms 238

4.1. Structure of the Fermi Hole and Exchange Potential 238

4.2. Structure of the Coulomb Hole and Correlation Potential 242

4.3. Ground-State Properties in the Pauli-Correlated Approximation 247

4.4. Ionization Potentials, Electron Affinities and Transition Energies 251

5. Conclusions and Future Work 255

References $\quad 257$

$\begin{array}{ll}\text { Index } & 261\end{array}$ 


\title{
F O R E W O R D
}

\author{
The rivers run into the sea, yet the sea is not full
}

Ecclesiastes

What is quantum chemistry? The straightforward answer is that it is what quantum chemists do. But it must be admitted, that in contrast to physicists and chemists, "quantum chemists" seem to be a rather ill-defined category of scientists. Quantum chemists are more or less physicists (basically theoreticians), more or less chemists, and by and large, computationists. But first and foremost, we, quantum chemists, are conscious beings.

We may safely guess that quantum chemistry was one of the first areas in the natural sciences to lie on the boundaries of many disciplines. We may certainly claim that quantum chemists were the first to use computers for really large scale calculations. The scope of the problems which quantum chemistry wishes to answer and which, by its unique nature, only quantum chemistry can answer is growing daily. Retrospectively we may guess that many of those problems meet a daily need, or are say, technical in some sense. The rest are fundamental or conceptual. The daily life of most quantum chemists is usually filled with grasping the more or less technical problems. But it is at least as important to devote some time to the other kind of problems whose solution will open up new perspectives for both quantum chemistry itself and for the natural sciences in general.

When chemistry developed in the nineteenth century, its explanatory systems began to separate from those of physics, as peculiarly chemical theories of molecular structure and of reactivity were conceived. By the early twentieth century attempts were made to heal this rift and perhaps the most influential of these was the creation of the electronic theory of valency.

However it was not until the development of quantum mechanics in the late nineteen- twenties that it became clear how a proper reconciliation should be effected. It is in this context that quantum chemistry rises as a subject of its own and 
gets a very special place among the natural sciences. Quantum chemistry provides the conceptual apparatus by means of which chemical explanation is tied to the explanatory scheme of quantum mechanics, one of the most fundamental of all physical theories.

Until the nineteen-fifties - even though important numerical work was certainly carried out also before that time - the chief contribution of quantum chemistry to chemistry was in terms of concepts like atomic and molecular orbitals, resonance, hybridisation and many others. Since about nineteen- sixty the development of computers has led to a change in emphasis. The conceptual apparatus has remained largely that of previous generations but thanks to large scale sophisticated quantum chemical computations, quantitatively accurate results have made it possible to assist in an effective way the understanding of many phenomena in chemistry, physics, and biology. There can be no doubt about the tremendous quantitative successes that quantum chemistry has had in the last three decades. However, it may not be out of place to have a look retrospectively at different angles and to ask: Whether time might not be ripe for making a paradigm shift in the nature of the concepts that are used, for breaching the decorum to say what has not yet been said before because it has seemed too trivial to bother with or too embarrassing to own up to, and for showing us something that had been there all the time that we had not seen for ourselves.

That was our idea to launch "Conceptual Trends in Quantum Chemistry". This former collection of essays of a number of active quantum chemists has been appeared under the identical title in the early 1994 . The present one has the same underlying idea, although it is slightly more specified by the purpose. We hope that both these

We are deeply indebted to the KLUWER Academic Publishers and personally, to Mrs. Wil Bruins and Dr. David J. Larner for all their interest and assistance in the preparation of this series. 